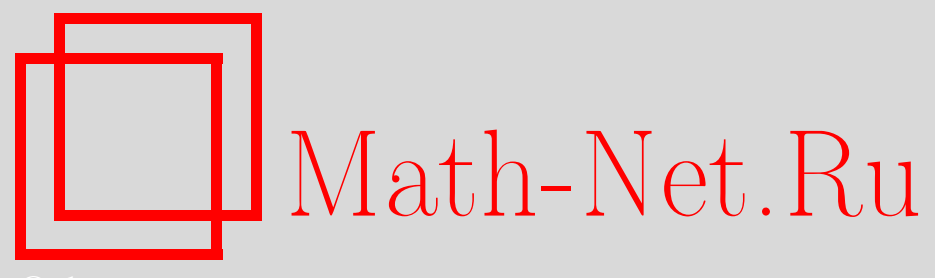

Б. А. Погорелов, М. А. Пудовкина, Натуральные метрики и их свойства. Ч. 1. Подметрики и надметрики, Матем. вопр. криптогр., 2011, том 2, выпуск 4, 49-74

DOI: https://doi.org/10.4213/mvk43

Использование Общероссийского математического портала Math-Net.Ru подразумевает, что вы прочитали и согласны с пользовательским соглашением http: //www . mathnet.ru/rus/agreement

Параметры загрузки:

IP: 54.198 .187 .58

26 апреля 2023 г., 15:14:05 


\title{
Натуральные метрики и их свойства. Ч. 1. Подметрики и надметрики
}

\author{
Б. А. Погорелов ${ }^{1)}$, М. А. Пудовкина ${ }^{2)}$ \\ 1) Академия криптографии Российской Федерации, Москва \\ 2) Национальный исследовательский ядерный университет (МИФИ), Москва
}

Получено 22.IV.2010

Найдены критерии того, что целочисленная функция $\mu: X \times X \rightarrow\{0$, $1, \ldots\}$, где $X-$ конечное множество, является метрикой. Вводятся понятия подметрики, надметрики, натуральной и канонической метрик. Проведена классификация метрик, принимающих не более 5 значений, описаны некоторые их подметрики и надметрики.

Ключевые слова: дискретные пространства, дискретные метрики, характеризация метрик

\section{Natural metrics and their properties. P. 1. Submetrics and overmetrics}

\author{
B. A. Pogorelov', M. A. Pudovkina ${ }^{2)}$ \\ ${ }^{1)}$ Academy of Cryptography of Russian Federation, Moscow \\ 2) National Nuclear Research University, Moscow
}

\begin{abstract}
Criteria for integer-valued function $\mu: X \times X \rightarrow\{0,1, \ldots\}$ to be a metric (where $X$ is a finite set) are given. Notions of submetric, overmetric, natural and canonical metrics are introduced. Classification of metrics admitting no more thann 5 values is constructed, some of their submetrics and overmetrics are described.
\end{abstract}

Key words: discrete spaces, discrete metrics, characterization of metrics

Citation: Mathematical Aspects of Cryptography, 2011, vol. 2, no. 4, pp. 49-74 (Russian). 


\section{§ 1. Введение}

В последние годы заметно возрос интерес к различным дискретным метрикам, в том числе и отличным от метрик Хемминга, Ли, Левенштейна. Необходимость в таких метриках возникает, в частности, в теории кодирования, теории дискретных функций, теории графов, комбинаторике, теории групп подстановок, при тестировании псевдослучайных последовательностей и в криптографии [1], [2], [3], [8], [9], [10].

Известно, что сплетение групп допускает два точных подстановочных представления: сплетение групп подстановок (подстановочное представление импримитивно) и экспоненцирование групп подстановок (группа может быть примитивной). В первом случае операции сплетения соответствует гомоморфизм импримитивной группы. Во втором случае таких гомоморфизмов нет, но возникает метрика, которую сохраняет экспоненцирование групп. В терминах экспоненцирования групп описывается группа изометрий метрики Хемминга. Эти факты говорят о том, что различные метрические соотношения могут заменять гомоморфизмы в случае примитивного действия групп подстановок, что является дополнительным стимулом изучения дискретных метрик.

В работе [5] при описании подсхем схемы Хемминга было введено понятие подметрики метрики Хемминга. В работах [5], [6] и [8] даны классификация и описание свойств групповых подметрик метрики Хемминга, т. е. метрик, соответствующих подсхемам схемы Хемминга или орбиталам надгрупп группы Джевонса. Однако интерес представляют и нехемминговы метрики, в том числе малой значности.

В этой первой части статьи в терминах метрических орбиталов приводятся критерии того, что функция является метрикой. Вводятся понятия натуральной метрики (т. е. метрики с множеством значений $0,1, \ldots, d$ ) и её обобщения - канонической метрики. Отметим, что в общем случае каноническая метрика может не являться натуральной. Обе они являются в определённом смысле минимальными. Затем в терминах подметрик, надметрик, натуральных метрик, канонических метрик осуществляется упорядочивание и первоначальная классификация целочисленных конечнозначных метрик, в том числе канонических метрик значности не более 5, а также некоторых классов подметрик и надметрик натуральных метрик.

В работе рассматриваются только конечные и целочисленные метрики.

\section{Основные обозначения}

- $\mathbb{N}\left(\mathbb{N}_{0}\right)$ - множество всех натуральных чисел (с нулём);

- $\chi_{t}$ - метрика Хемминга на $t$-мерном векторном пространстве $V_{t}=V_{t}(2)$ над полем $G F(2)$; 
- $\varepsilon_{j, t} \in V_{t}, \varepsilon_{j, t}=(0, \ldots, 0,1,0, \ldots, 0), j \in\{1, \ldots, t\}$;

- $\left(A_{1}, \ldots, A_{m}\right)$ - разбиение множества $A$ на $m$ непустых попарно не пересекающихся подмножеств $A_{1}, \ldots, A_{m}$;

- $I=\{(x, x) \mid x \in X\}$;

- $\Delta_{t}$ - множество всех векторов из $V_{n}$ веса Хемминга $t \in\{1, \ldots, n\}$;

- $\overline{\mathbf{X}}^{(2)}$ - множество всех таких разбиений множества $(X \times X) \backslash I$, что каждый блок разбиения является симметричным подмножеством множества $(X \times X) \backslash I$;

- $K(X)=\left\{\mu_{\overline{\mathrm{A}}} \mid \overline{\mathrm{A}} \in \overline{\mathbf{X}}^{(2)}\right\}-$ множество всех канонических метрик, соответствующих разбиениям $\overline{\mathrm{A}} \in \overline{\mathbf{X}}^{(2)}$;

- $\lceil\gamma\rceil$ - наименьшее целое число $\gamma^{\prime}$ такое, что $\gamma \leq \gamma^{\prime}$;

- $\mathrm{N}(X)$ - множество всех натуральных метрик на множестве $X$;

Если $\mu-(d+1)$-значная метрика, то:

- $\quad \mu^{i \uparrow}-(d+1+i)$-значная надметрика метрики $\mu$,

- $\quad \mu_{j}^{i \uparrow}-j$-я $(d+1+i)$-значная надметрика метрики $\mu$,

- $\left\{\mu^{i \uparrow}\right\}$ - множество $(d+1+i)$-значных надметрик метрики $\mu$,

- $\mu^{i \downarrow}-(d+1-i)$-значная подметрика метрики $\mu$;

- $\mu_{j}^{i \downarrow}-j$-я $(d+1-i)$-значная подметрика метрики $\mu$,

- $\left\{\mu^{i \downarrow}\right\}$ - множество $(d+1-i)$-значных подметрик метрики $\mu$,

- $\quad \mu^{\downarrow}=\mu^{1 \downarrow},\left\{\mu^{\downarrow}\right\}=\left\{\mu^{1 \downarrow}\right\}, \mu^{\uparrow}=\mu^{1 \uparrow},\left\{\mu^{\uparrow}\right\}=\left\{\mu^{1 \uparrow}\right\}$.

Если размерность $t$ пространства $V_{t}$ понятна из контекста, то у метрики $\chi_{t}$ и вектора $\varepsilon_{j, t}$ символ « $t »$ будем опускать.

При задании метрики будем опускать всегда выполняющееся условие $\mu(\alpha, \alpha)=0$.

\section{\$ 2. Общие свойства конечных целочисленных метрик}

Для любых подмножеств $\bar{A}, \bar{B} \subseteq X \times X$ обозначим

$$
\begin{gathered}
\bar{A} \circ \bar{B}=\{(\alpha, \beta) \mid \exists \gamma \in X:(\alpha, \gamma) \in \bar{A},(\gamma, \beta) \in \bar{B}\}, \\
\bar{A} * \bar{B}=\bar{A} \circ \bar{B} \bigcup \bar{B} \circ \bar{A} .
\end{gathered}
$$


Пусть $X-$ произвольное конечное множество. Назовём множество (блок) $\bar{A} \subseteq X \times X$ симметричным, если $\left(\alpha_{1}, \alpha_{2}\right) \in \bar{A}$ тогда и только тогда, когда $\left(\alpha_{2}, \alpha_{1}\right) \in \bar{A}$. Блок $I$ назовём тривиальным симметричным. Пусть $\bar{X}^{(2)}-$ множество всех симметричных подмножеств множества $(X \times X) \backslash I$. Для любого $\bar{Y} \in \bar{X}^{(2)}$ положим

$$
\overline{\mathbf{Y}}^{(2)}=\left\{\left.\left(\bar{A}_{1}, \ldots, \bar{A}_{l}\right)\right|_{i=1} ^{l} \bar{A}_{i}=\bar{Y}, \bar{A}_{1}, \ldots, A_{l} \in \bar{X}^{(2)}, \bar{A}_{i} \cap \bar{A}_{j}=\varnothing, 1 \leq i<j \leq l, l \in \mathbb{N}\right\},
$$

т. е. $\overline{\mathbf{Y}}^{(2)}$ - множество всех таких разбиений множества $\bar{Y}$, что каждый из блоков разбиения является симметричным подмножеством из множества $X \times X$.

Для набора чисел $v=\left(v_{1}, \ldots, v_{d}\right) \in \mathbb{N}^{d}$ и числа $r \in \mathbb{N}$ обозначим

$$
\begin{gathered}
P_{v}(r)=\left\{\{i, j\} \subseteq\{1, \ldots, d\} \mid v_{i}+v_{j}=r\right\}, \\
C_{v}(r)=\left\{c \in\{1, \ldots, d\} \mid v_{c} \leq r\right\} .
\end{gathered}
$$

Пусть $d \in \mathbb{N}$ и функция $\mu: X \times X \rightarrow \mathbb{N}$, определенная условиями

$$
\mu\left(\alpha, \alpha^{\prime}\right)=v_{i} \text { при }\left(\alpha, \alpha^{\prime}\right) \in \bar{A}_{i}, i \in\{1, \ldots, d\},
$$

где $\left(\bar{A}_{1}, \ldots, \bar{A}_{d}\right) \in \overline{\mathbf{X}}^{(2)}$, является метрикой. Тогда множества $\bar{A}_{1}, \ldots, \bar{A}_{d}$ будем называть метрическими орбиталами, а число $(d+1)$ - значностью метрики.

Tеорема 2.1. Пусть $d \geq 2,\left(\bar{A}_{1}, \ldots, \bar{A}_{d}\right) \in \overline{\mathbf{X}}^{(2)} u v_{1}, \ldots, v_{d} \in \mathbb{N}, \quad v_{1}<\ldots<v_{d}$. Функиия $\mu: X \times X \rightarrow \mathbb{N}_{0}$, заданная условиями

$$
\mu\left(\alpha, \alpha^{\prime}\right)=v_{i}, \text { если }\left(\alpha, \alpha^{\prime}\right) \in A_{i}, i \in\{1, \ldots, d\},
$$

является метрикой тогда и только тогда, когда для всех $r \in\left\{v_{1}+1, \ldots, v_{d}\right\}$ справедливы включения

$$
\bigcup_{\{i, j\} \in P_{v}(r)} \bar{A}_{i} * \bar{A}_{j} \subseteq \bigcup_{c \in C_{v}(r)} \bar{A}_{c} .
$$

Доказательство. (Необходимость.) Из неравенства треугольника следует, что для каждого числа $r \in\left\{v_{1}+1, v_{1}+2, \ldots, v_{d}\right\}$ и любой пары $\{i, j\} \in P(r)$ должно выполняться включение

$$
\bar{A}_{i} * \bar{A}_{j} \subseteq \bigcup_{c \in C_{v}(r)} \bar{A}_{c} .
$$


Отсюда получаем, что

$$
\bigcup_{\{i, j\} \in P_{v}(r)} \bar{A}_{i} * \bar{A}_{j} \subseteq \bigcup_{c \in C_{v}(r)} \bar{A}_{c} \text { для каждого } r \in\left\{v_{1}+1, v_{1}+2, \ldots, v_{d}\right\} .
$$

(Достаточность.) Очевидно, что достаточно проверить справедливость неравенства треугольника для всех пар $(\alpha, \beta),(\alpha, \gamma),(\gamma, \beta) \in(X \times X) \backslash I$.

Зафиксируем произвольные $l_{1}, l_{2} \in\{1, \ldots, d\}$ и рассмотрим произвольные пары $(\alpha, \gamma) \in \bar{A}_{l_{1}},(\gamma, \beta) \in \bar{A}_{l_{2}}$.

Если $v_{l_{1}}+v_{l_{2}} \geq v_{d}$, то неравенство треугольника

$$
\mu(\alpha, \beta) \leq v_{d} \leq \mu(\alpha, \gamma)+\mu(\gamma, \beta)
$$

справедливо.

Обозначим $v(i, j)=v_{i}+v_{j}$ для всех $i, j \in\{1, \ldots, d\}$. Пусть $v_{l_{1}}+v_{l_{2}}<v_{d}$. В силу включения (2.1) имеем

$$
\bar{A}_{l_{1}} \circ \bar{A}_{l_{2}} \subseteq \bigcup_{c=1}^{v\left(l_{1}, l_{2}\right)} \bar{A}_{c}
$$

Следовательно,

$$
(\alpha, \beta) \in \bigcup_{c=1}^{v\left(l_{1}, l_{2}\right)} \bar{A}_{c}
$$

Значит,

$$
v_{v\left(l_{1}, l_{2}\right)} \leq v_{l_{1}}+v_{l_{2}},
$$

что означает справедливость неравенства треугольника

$$
\mu(\alpha, \beta) \leq v_{l_{1}}+v_{l_{2}}=\mu(\alpha, \gamma)+\mu(\gamma, \beta) .
$$

Теорема доказана.

Отметим, что существуют метрики, у которых все ненулевые значения нечётны. Например, рассмотрим произвольное разбиение $\left(\bar{A}_{1}, \ldots, \bar{A}_{d}\right) \in \overline{\mathbf{X}}^{(2)}$; пусть $v_{1}$ - произвольное нечётное число, $v_{i+1}=2 v_{i}+1$ для $i=1, \ldots, d-1$. Тогда выполнены условия теоремы 2.1 и все ненулевые значения соответствующей метрики являются нечётными.

Конечные метрики, принимающие все значения из множества $\{0, \ldots, d\}$ для некоторого натурального числа $d \geq 2$, можно считать в некотором смысле минимальными данной значности и рассматривать как «строительные блоки» для задания других конечных целочисленных метрик. Приведём критерий, позволяющий описать такие метрики. 
Следствие 2.2. Пусть выполнены условия теоремы 2.1, $\bar{A}_{0}=I, \bar{H}_{j}=\bigcup_{i=0}^{j} \bar{A}_{i}$, где $j=1, \ldots, d, u v_{i}=i$ для всех $i \in\{1, \ldots, d\}$. Функиия $\mu: X \times X \rightarrow \mathbb{N}_{0}$, заданная условиями

$$
\mu\left(\alpha, \alpha^{\prime}\right)=i, \text { если }\left(\alpha, \alpha^{\prime}\right) \in \bar{A}_{i}, i \in\{1, \ldots, d\},
$$

является метрикой тогда и только тогда, когда для всех $j \in\{2, \ldots, d\}$ справедливо включение

$$
\bigcup_{i=1}^{j-1}\left(\bar{A}_{i} \circ \bar{A}_{j-i}\right) \subseteq \bar{H}_{j}
$$

Доказательство следует из теоремы 2.1 и справедливости для всех $r \in\{1, \ldots, d\}$ равенств $P_{v}(r)=\{\{i, r-i\} \subseteq\{1, \ldots, d\}\}, C_{v}(r)=\{1, \ldots, r\}$.

Метрику вида (2.2) назовём натуральной. Обозначим через $\mathrm{N}(X)$ множество всех натуральных метрик на множестве $X$.

Пусть $(X, \rho)$ - метрическое пространство с метрикой $\rho: X^{n} \rightarrow \mathbb{R}_{0}$, $\alpha=\left(\alpha_{1}, \ldots, \alpha_{n}\right), \beta=\left(\beta_{1}, \ldots, \beta_{n}\right) \in X^{n}$. На множестве $X^{n}$ в ряде случаев (в теории кодирования, функциональном и математическом анализе) рассматриваются следующие метрики:

$$
\begin{gathered}
\rho_{1}(\alpha, \beta)=\sum_{i=1}^{n} \rho\left(\alpha_{i}, \beta_{i}\right), \\
\rho_{2}(\alpha, \beta)=\max \left\{\rho\left(\alpha_{i}, \beta_{i}\right) \mid i \in\{1, \ldots, n\}\right\}, \\
\rho_{3}(\alpha, \beta)=\left(\sum_{i=1}^{n} \rho\left(\alpha_{i}, \beta_{i}\right)^{p}\right)^{1 / p}, p \in \mathbb{N} .
\end{gathered}
$$

Можно также рассмотреть целочисленные метрики

$$
\rho_{k}^{\prime}(\alpha, \beta)=\left\lceil\rho_{k}(\alpha, \beta)\right\rceil, \quad k=1,2,3 .
$$

Нетрудно показать, если $\rho-$ натуральная метрика, то $\rho_{1}^{\prime}, \rho_{2}^{\prime}$ и $\rho_{3}^{\prime}-$ также натуральные метрики.

Приведём переформулировку следствия 2.2 для метрик, инвариантных относительно группы сдвигов пространства $V_{n}$. К таким метрикам относится, например, метрика Хемминга.

Для подмножеств $A, B \subseteq X$ положим $A+B=\{\alpha+\beta \mid \alpha \in A, \beta \in B\}$. 
Следствие 2.3. Пусть $\left(A_{1}, \ldots, A_{d}\right)$ - разбиение $V_{n} \backslash \overrightarrow{0}, \quad d \geq 2$, $\bar{H}_{j}=\{\overrightarrow{0}\} \cup\left(\bigcup_{i=1}^{j} A_{i}\right)$. Функиия $\mu: V_{n} \times V_{n} \rightarrow\{0, \ldots, d\}$, заданная условиями

$$
\mu\left(\alpha, \alpha^{\prime}\right)=i, \text { если } \alpha+\alpha^{\prime} \in A_{i}, i \in\{1, \ldots, d\},
$$

тогда и только тогда является метрикой, когда для всех $j \in\{2, \ldots, d\}$ справедливо включение

$$
\bigcup_{i=1}^{j-1}\left(A_{i}+A_{j-i}\right) \subseteq H_{j}
$$

Доказательство непосредственно следует из теоремы 2.1.

Покажем, что каждому разбиению $\left(\bar{A}_{1}, \ldots, \bar{A}_{d}\right) \in \overline{\mathbf{X}}^{(2)}$ соответствует конечная целочисленная метрика.

Следствие 2.4. Пусть $d \geq 2 \quad u\left(\bar{A}_{1}, \ldots, \bar{A}_{d}\right) \in \overline{\mathbf{X}}^{(2)}$. Тогда сущзествуют такие числа $v_{1}, \ldots, v_{d} \in \mathbb{N}$, где $v_{1}<\ldots<v_{d}$, что функция

$$
\mu\left(\alpha, \alpha^{\prime}\right)=v_{i}, \text { если }\left(\alpha, \alpha^{\prime}\right) \in \bar{A}_{i}, i \in\{1 \ldots, d\},
$$

является метрикой. В частности, если $v_{i}=d+i-2$ для всех $i \in\{1, \ldots, d\}$, то функиия $\mu-$ метрика.

Доказательство следует из теоремы 2.1.

Согласно теореме 2.1, множество метрик, соответствующих разбиению $\left(\bar{A}_{1}, \ldots, \bar{A}_{d}\right) \in \overline{\mathbf{X}}^{(2)}, d \geq 1$, является счётным. Поэтому представляет интерес среди них выделить «наименьшую» возможную метрику, которую можно считать в некотором смысле канонической.

Пусть $\quad d \geq 1, \quad \overline{\mathrm{A}}=\left(\bar{A}_{1}, \ldots, \bar{A}_{d}\right) \in \overline{\mathbf{X}}^{(2)}, \quad v=\left(v_{1}, \ldots, v_{d}\right) \in \mathbb{N}^{d} \quad$ и $\quad \mu_{\mathrm{A}}^{v}: X \times$ $\times X \rightarrow\{1, \ldots, d\}$, где $\mu_{\mathrm{A}}^{v}\left(\alpha, \alpha^{\prime}\right)=v_{i}$, если $\left(\alpha, \alpha^{\prime}\right) \in \bar{A}_{i}, i \in\{1, \ldots, d\}$.

Обозначим через $\mathrm{M}(\overline{\mathrm{A}})$ множество всех функций $\mu_{\mathrm{A}}^{v}$, являющихся метриками. Если $I(B)$ - индикатор выполнения условия $B$, то тривиальная метрика $\mu\left(\alpha, \alpha^{\prime}\right)=I\left(\alpha \neq \alpha^{\prime}\right)$ также принадлежит множеству $\mathrm{M}(\overline{\mathrm{A}})$. Из теоремы 2.1 следует, что $\mu_{\mathrm{A}}^{v} \in \mathrm{M}(\overline{\mathrm{A}})$ тогда и только тогда, когда для всех $r=2, \ldots, v_{d}$

$$
\bigcup_{\{i, j\} \in P_{v}(r)} \bar{A}_{i} * \bar{A}_{j} \subseteq \bigcup_{c \in C_{v}(r)} \bar{A}_{c} .
$$


Для натурального числа $d \geq 1$ на множестве

$$
\mathbb{N}^{(d)}=\left\{\left(v_{1}, \ldots, v_{d}\right) \in \mathbb{N}^{d} \mid v_{1}<\ldots<v_{d}\right\}
$$

зададим отношение линейного (лексикографического) порядка $\preceq$, полагая $v=\left(v_{1}, \ldots, v_{d}\right) \preceq v^{\prime}=\left(v_{1}{ }^{\prime}, \ldots, v_{d}{ }^{\prime}\right)$, если $v=v^{\prime}$ или существует такое число $t \in\{1, \ldots, d\}$, что $v_{i}=v_{i}^{\prime}$ для всех $i \in\{1, \ldots, t-1\}$ и $v_{t}<v_{t}^{\prime}$. Если $v \preceq v^{\prime}$ и $v \neq v^{\prime}$, то будем использовать обозначение $v \prec v^{\prime}$.

Для заданного разбиения $\overline{\mathrm{A}}=\left(\bar{A}_{1}, \ldots, \bar{A}_{d}\right) \in \overline{\mathbf{X}}^{(2)}$ метрику $\mu_{\mathrm{A}}^{v} \in \mathrm{M}(\overline{\mathrm{A}})$ назовём канонической, если $v \preceq v^{\prime}$ для каждой метрики $\mu_{\mathrm{A}}^{v^{\prime}} \in \mathrm{M}(\overline{\mathrm{A}}), v^{\prime} \in \mathbb{N}^{(d)}$.

Из утверждения 2.5 (см. ниже) будет следовать единственность канонической метрики. Обозначим каноническую метрику разбиения $\overline{\mathrm{A}} \in \overline{\mathbf{X}}^{(2)}$ через $\mu_{\mathrm{A}}$. Ясно, что каждой канонической метрике $\mu_{\mathrm{A}} \in \mathrm{M}(\overline{\mathrm{A}})$ однозначно соответствует такой набор $\left(v_{1}, \ldots, v_{d}\right) \in \mathbb{N}^{(d)}$, что $\mu_{\mathrm{A}}^{v}=\mu_{\mathrm{A}}$. Кроме того, каждая натуральная метрика является канонической.

Пусть $\mathrm{K}(X)$ - множество канонических метрик, соответствующих всем нетривиальным разбиениям $\overline{\mathrm{A}} \in \overline{\mathbf{X}}^{(2)}$.

Следующее утверждение существенно упрощает применение критерия, приведённого в теореме 2.1, для нахождения канонической метрики $\mu_{\mathrm{A}}$ и метрик из множества $\mathrm{K}(X)$ для произвольного разбиения $\mathrm{A} \in \overline{\mathbf{X}}^{(2)}$, сводя проблему к решению системы линейных целочисленных неравенств.

Утверждение 2.5. Пусть $d \geq 2, \quad\left(\bar{A}_{1}, \ldots, \bar{A}_{d}\right) \in \overline{\mathbf{X}}^{(2)}, \quad v_{1}, \ldots, v_{d} \in \mathbb{N}$, $v_{1}<\ldots<v_{d}, u$

$$
b_{i j}=\max \left\{t \in\{1, \ldots, d\} \mid \bar{A}_{i} \circ \bar{A}_{j} \cap \bar{A}_{t} \neq \varnothing\right\}
$$

для всех $i, j \in\{1, \ldots, d\}$. Функция $\mu: X \times X \rightarrow \mathbb{N}_{0}$, заданная условиями

$$
\mu\left(\alpha, \alpha^{\prime}\right)=v_{i}, \text { если }\left(\alpha, \alpha^{\prime}\right) \in \bar{A}_{i}, i \in\{1, \ldots, d\},
$$

является метрикой тогда и только тогда, когда $v_{1}, \ldots, v_{d}$ удовлетворяют системе неравенств

$$
v_{i}+v_{j} \geq v_{b_{i j}}, i, j \in\{1, \ldots, d\} .
$$

Доказательство. (Необходимость.) Пусть $\mu$ - метрика. Из теоремы 2.1 следует справедливость включения

$$
\bigcup_{\{i, j\} \in P_{v}(r)} \bar{A}_{i} * \bar{A}_{j} \subseteq \bigcup_{c \in C_{v}(r)} \bar{A}_{c} .
$$


для всех $r \in\left\{2, \ldots, v_{d}\right\}$. Если $r \in\left\{v_{1}+1, \ldots, v_{d}\right\} \backslash\left\{v_{1}, v_{2}, \ldots, v_{d}\right\}$, то для каждого множества $\{i, j\} \in P_{v}(r) \quad$ имеем $b_{i j} \leq \max \left\{c \mid c \in C_{v}(r)\right\} \quad$ и $v_{b_{i j}}<r$. Значит, $v_{i}+v_{j}>v_{b_{i j}}$.

Если $r \in\left\{v_{1}, \ldots, v_{d}\right\}$, то для каждого множества $\{i, j\} \in P_{v}(r)$ из соотношений

$$
v_{b_{i j}} \leq r, \quad \bar{A}_{i} \circ \bar{A}_{j} \subseteq \bigcup_{c=0}^{b_{i j}} \bar{A}_{c} \subseteq \bigcup_{c=0}^{r} \bar{A}_{c}
$$

следует, что $v_{b_{i j}} \leq v_{i}+v_{j}$.

(Достаточность.) Пусть числа $v_{1}, \ldots, v_{d}$ Удовлетворяют неравенствам

$$
v_{i}+v_{j} \geq v_{b_{i j}}, i, j \in\{1, \ldots, d\} .
$$

Покажем, что $\mu$ метрика. Зафиксируем произвольные числа $i, j \in\{1, \ldots, d\}$ и рассмотрим произвольную пару $(\alpha, \beta) \in \bar{A}_{i},(\beta, \gamma) \in \bar{A}_{j}$. Тогда из определения $b_{i j}$ получаем $(\alpha, \gamma) \subseteq \bigcup_{c=0}^{b_{i j}} \bar{A}_{c}$. Значит,

$$
\mu(\alpha, \beta)+\mu(\beta, \gamma)=v_{i}+v_{j} \geq v_{b_{i j}} \geq \mu(\alpha, \gamma) \text {. }
$$

Утверждение доказано.

Отметим, что наименьшее (относительного линейного порядка $\preceq$ ) решение $\left(v_{1}^{\prime}, \ldots, v_{d}^{\prime}\right) \in \mathbb{N}^{(d)}$ системы неравенств (2.3) задаёт каноническую метрику $\mu_{\mathrm{A}}^{v^{\prime}}$.

Пусть $\left(\bar{A}_{1}, \ldots, \bar{A}_{d}\right) \in \overline{\mathbf{X}}^{(2)}$. Для $(d+1)$-значной метрики

$$
\mu_{\mathrm{A}}^{v}\left(\alpha, \alpha^{\prime}\right)=v_{i}, \text { если }\left(\alpha, \alpha^{\prime}\right) \in \bar{A}_{i}, i \in\{1, \ldots, d\},
$$

рассмотрим $(d \times d)$-матрицу $B_{\mathrm{A}}=\left(b_{i j}\right)$, где $b_{i j}=\max \left\{t \in\{1, \ldots, d\} \mid \bar{A}_{i} \circ \bar{A}_{j} \cap \bar{A}_{t} \neq \varnothing\right\}$, $i, j \in\{1, \ldots, d\}$. Очевидно, что матрица $B_{\text {А }}$ симметричная. Каждой такой матрице можно сопоставить граф. Это позволяет каждой канонической метрике и, соответственно, каждому разбиению $\overline{\mathrm{A}}=\left(\bar{A}_{1}, \ldots, \bar{A}_{d}\right) \in \overline{\mathbf{X}}^{(2)}$ сопоставить граф и рассматривать свойства этого графа и свойства этой метрики. 
Из утверждения 2.5 следует, что при заданном разбиении $\overline{\mathrm{A}}=$ $=\left(\bar{A}_{1}, \ldots, \bar{A}_{d}\right) \in \overline{\mathbf{X}}^{(2)}$ для нахождения канонической метрики $\mu_{\mathrm{A}}$ достаточно найти наименьшее (относительного линейного порядка $\preceq$ ) решение из $\mathbb{N}^{(d)}$ системы неравенств (2.3), а все метрики из множества $\mathrm{M}(\overline{\mathrm{A}})$ однозначно задаются решениями системы неравенств (2.3).

Следствие 2.6. Пусть $d \geq 2,\left(\bar{A}_{1}, \ldots, \bar{A}_{d}\right) \in \overline{\mathbf{X}}^{(2)} u v_{1}, \ldots, v_{d} \in \mathbb{N}, v_{1}<\ldots<v_{d}$.

(1) При любых таких числах $l, d_{1}, \ldots, d_{l}, t_{1}, \ldots, t_{l} \in \mathbb{N}$, что $d=d_{1}+\ldots+d_{l}$, $u t_{j}+d_{j}<t_{j+1}$ для всех $j \in\{1, \ldots, l-1\}$, функщия $\mu: X \times X \rightarrow \mathbb{N}_{0}$, заданная условиями

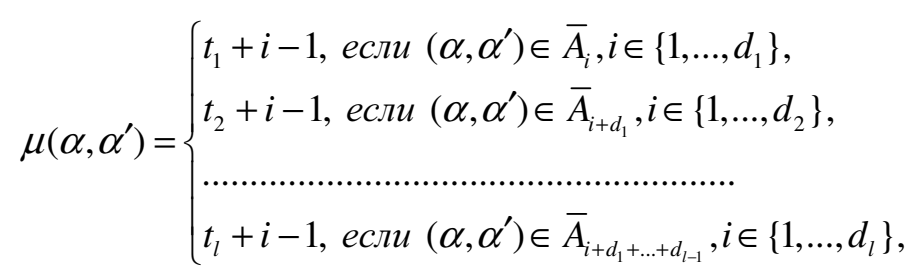

является метрикой тогда и только тогда, когда для всех $c_{1}, c_{2} \in\{1, \ldots, l\}$ справедливы неравенства

$$
\geq \max \left\{\begin{array}{l|l}
t_{c_{1}}+t_{c_{2}} \geq \\
\left.t_{c_{3}}+i_{3}-i_{1}-i_{2}+1 \mid \begin{array}{l}
j_{w}=\sum_{p=1}^{c_{w}-1} d_{p}+i_{w}, i_{w} \in\left\{1, \ldots, d_{c_{w}}\right\}, w \in\{1,2\}, \\
b_{j_{1} j_{2}}=\sum_{p=1}^{c_{3}-1} d_{p}+i_{3}, j_{3} \in\left\{1, \ldots, d_{c}\right\}, c_{3} \in\{1, \ldots, l\}
\end{array}\right\} .
\end{array}\right.
$$

(2) При $d \geq 2, \overline{\mathrm{A}}=\left(\bar{A}_{1}, \ldots, \bar{A}_{d}\right) \in \overline{\mathbf{X}}^{(2)}$ существует такое наименьщее число $t \in\{0, \ldots, d-1\}$, равное $t=\max \left\{b_{i j}-(i+j-1) \mid i, j \in\{1, \ldots, d\}\right\}$, что функция $\mu^{(t)}: X \times X \rightarrow \mathbb{N}_{0}$, заданная условиями

$$
\mu^{(t)}\left(\alpha, \alpha^{\prime}\right)=t+i-1, \text { если }\left(\alpha, \alpha^{\prime}\right) \in \bar{A}_{i}, i \in\{1, \ldots, d\},
$$

является метрикой. Кроме того, для любого $t^{\prime} \geq t$ функиия $\mu^{\left(t^{\prime}\right)}$ является метрикой.

Доказательство. (1) Согласно утверждению 2.5, функция $\mu$ является метрикой тогда и только тогда, когда для всех $i_{1}, i_{2} \in\{1, \ldots, d\}$ справедливы неравенства

$$
v_{i_{1}}+v_{i_{2}} \geq v_{b_{i_{12}}} .
$$


Зафиксируем произвольные числа $c_{1}, c_{2} \in\{1, \ldots, l\}, \quad i_{1} \in\left\{1, \ldots, d_{c_{1}}\right\}$, $i_{2} \in\left\{1, \ldots, d_{c_{2}}\right\}$ и положим

$$
v_{i_{w}}=t_{c_{w}}+i_{w}-1, \quad j_{w}=\sum_{p=1}^{c_{w}-1} d_{p}+i_{w}, w \in\{1,2\}
$$

Тогда

$$
b_{j_{1} j_{2}}=\sum_{p=1}^{c_{3}-1} d_{p}+i_{3}, \quad v_{i_{w}}=t_{c_{3}}+i_{3}-1
$$

где $i_{3} \in\left\{1, \ldots, d_{c_{3}}\right\}, c_{3} \in\{1, \ldots, l\}$. В этом случае система (2.3) принимает вид

$$
t_{c_{1}}+t_{c_{2}} \geq t_{c_{3}}-\left(i_{1}+i_{2}-1\right)+i_{3}
$$

(2) Положим $\quad v_{i}=t+i-1, \quad i \in\{1, \ldots, d\}, v_{b_{i_{1} i_{2}}}=b_{i_{1} i_{2}}+t-1 \quad$ для $\quad$ всех $i_{1}, i_{2} \in\{1, \ldots, d\}$ и подставим в систему (2.3). Имеем

$$
t-1+i+j \geq b_{i j}
$$

Следовательно, если

$$
t=\max \left\{b_{i j}-(i+j-1) \mid i, j \in\{1, \ldots, d\}\right\},
$$

то система (2.3) справедлива для всех $v_{1}, \ldots, v_{d}$. Значит, $t=\max \left\{b_{i j}-(i+j-1) \mid i, j \in\{1, \ldots, d\}\right\}$ - наименьшее число, при котором функция $\mu^{(t)}$ - метрика. Кроме того, для любого $t^{\prime} \geq t$ функция $\mu^{\left(t^{\prime}\right)}$ является метрикой. Следствие доказано.

Назовём метрику вида (2.4) кусочно-линейной, а метрику вида (2.5) линейной. Из следствия 2.6 получаем, что каждому разбиению $\overline{\mathrm{A}} \in \overline{\mathbf{X}}^{(2)}$ соответствует счётное множество кусочно-линейных метрик и единственная линейная метрика, минимальная относительно частичного порядка $\preceq$.

Приведём классификацию канонических 4-значных и 5-значных метрик (т. е. $d \leq 4)$ ). Нетрудно описать подобным образом также все канонические 6-значные, 7-значные метрики и т. д. Получающиеся при этом числа разных 
подслучаев и соответствующих им метрик быстро возрастают. Классификация содержится в следующем более общем результате.

Утверждение 2.7. Пусть $d \geq 2,\left(\bar{A}_{1}, \ldots, \bar{A}_{d}\right) \in \overline{\mathbf{X}}^{(2)}$. Тогда канонические метрики задаются следующим образом.

1. Если выполнено одно из условий:

a) $d \geq 2, b_{11}=d$,

б) $d \geq 4, b_{11}=d-3, b_{12}=d$,

mo

$$
\mu^{(1)}\left(\alpha, \alpha^{\prime}\right)=d+i-2, \text { если }\left(\alpha, \alpha^{\prime}\right) \in \bar{A}_{i}, i \in\{1, \ldots, d\} .
$$

2. Если выполнено одно из условий:

a) $d \geq 3, b_{11}=d-1$,

б) $d \geq 3, b_{11}=d-2, \quad b_{12}=d$,

в) $d \geq 4, b_{11}=d-3, b_{12} \leq d-2, b_{13}=d$,

г) $d \geq 4, b_{11}=d-3, b_{12}=d-1$,

mo

$$
\mu^{(2)}\left(\alpha, \alpha^{\prime}\right)=d+i-3, \text { если }\left(\alpha, \alpha^{\prime}\right) \in \bar{A}_{i}, i \in\{1, \ldots, d\} .
$$

3. Если выполнено одно из условий:

a) $d \geq 3, b_{11}=d-2$,

б) $d \geq 4, b_{11}=d-3, b_{12} \leq d-2, b_{13}=d$,

в) $d \geq 4, b_{11}=d-3, b_{12}=d-1$,

mo

$$
\mu^{(3)}\left(\alpha, \alpha^{\prime}\right)=d+i-4, \text { если }\left(\alpha, \alpha^{\prime}\right) \in \bar{A}_{i}, i \in\{1, \ldots, d\} .
$$

4. Если $d \geq 4, b_{11}=d-3, b_{12} \leq d-2, b_{13} \leq d-1 b_{22}=d$, то

$$
\mu^{(4)}\left(\alpha, \alpha^{\prime}\right)=\left\{\begin{array}{l}
d-3, \text { если }\left(\alpha, \alpha^{\prime}\right) \in \bar{A}_{1}, \\
d+i-3, \text { если }\left(\alpha, \alpha^{\prime}\right) \in \bar{A}_{i}, i \in\{2, \ldots, d\} .
\end{array}\right.
$$

Доказательство имеет технический характер и проводится непосредственной проверкой выполнения системы неравенств (2.3).

Для формулировки некоторых свойств канонических метрик нам понадобятся следующие обозначения. Рассмотрим множество

$$
\mathrm{K}_{\left(v_{1}, \ldots, v_{d}\right)}(X)=\left\{\mu_{\mathrm{A}} \in \mathrm{K}(X) \mid \mu_{\mathrm{A}}^{v}=\mu_{\mathrm{A}}, \overline{\mathrm{A}} \in \overline{\mathbf{X}}^{(2)}\right\}
$$


для произвольного набора $v=\left(v_{1}, \ldots, v_{d}\right) \in \mathbb{N}^{(d)}, d \in \mathbb{N}, 1 \leq d \leq C_{|X|}^{2}$. Обозначим

$$
\begin{gathered}
\Upsilon_{1}=\{1\}, \\
\Upsilon_{d}=\left\{\left(v_{1}, \ldots, v_{d}\right) \in \mathbb{N}^{d} \mid 1 \leq v_{1}<\ldots<v_{d} \leq 2(d-1), \mathrm{K}_{\left(v_{1}, \ldots, v_{d}\right)}(X) \neq \varnothing\right\}, \\
\Upsilon=\bigcup_{d \in\left\{1, \ldots, C_{|\mathbb{X}|}^{2}\right\}} \Upsilon_{d} .
\end{gathered}
$$

Отметим, что множество $\mathrm{K}_{(1)}(X)$ содержит только тривиальную метрику.

Ниже будет показано, что каждому разбиению $\left(\bar{A}_{1}, \ldots, \bar{A}_{d}\right) \in \overline{\mathbf{X}}^{(2)}, d \geq 2$, соответствуют набор чисел $v \in \Upsilon_{d}$ и каноническая метрика, для которой $\mu_{\mathrm{A}}^{v}=\mu_{\mathrm{A}}$, т. е. решения неравенства (2.3) достаточно искать среди элементов множества $\Upsilon_{d}$.

Лемма 2.8. Пусть $d \geq 2,\left(\bar{A}_{1}, \ldots, \bar{A}_{d}\right) \in \overline{\mathbf{X}}^{(2)}$.

1. Если $b_{11}=t, t \in\{1, \ldots, d\}$, и $\mu_{\mathrm{A}}^{v} \in \mathrm{K}(X)$, то $v_{1} \geq t-1$.

2. Если $b_{i, t-i}=t$ для любых $t \in\{1, \ldots, d\}, i \in\{1, \ldots, t-1\}$, то каноническая метрика задана равенствами $\mu\left(\alpha, \alpha^{\prime}\right)=i$, если $\left(\alpha, \alpha^{\prime}\right) \in \bar{A}_{i}, i \in\{1, \ldots, d\}$.

3. Если $\mu_{\mathrm{A}}^{v} \in \mathrm{K}(X)$, то $v_{1} \leq d-1$ и $v_{i} \leq 2(d-1)$ для всех $i \in\{2, \ldots, d\}$.

4. Справедливы соотношения $\mathrm{K}(X)=\bigcup_{v \in \Upsilon} \mathrm{K}_{v}(X)$ и $\mathrm{K}_{v}(X) \cap \mathrm{K}_{v^{\prime}}(X)=\varnothing$ для всех $v \neq v^{\prime}, v, v^{\prime} \in \Upsilon$.

Доказательство. 1 . Так как $v_{1}+v_{1} \geq v_{t}$ и $v_{t} \geq v_{1}+t-1$, то $v_{1} \geq t-1$.

2. Доказательство следует из утверждения 2.5.

3. Из следствия 2.4 получаем, что если $v_{i}^{\prime}=d+i-2$ для всех $i \in\{1, \ldots, d\}$, то $\mu_{\mathrm{A}}^{v^{\prime}} \in \mathrm{M}(\overline{\mathrm{A}})$ для произвольного $\overline{\mathrm{A}} \in \overline{\mathbf{X}}^{(2)}$. Ясно, что в этом случае $v_{i}^{\prime}$ удовлетворяют неравенствам $v_{1}^{\prime} \leq d-1, \quad v_{i}^{\prime} \leq 2(d-1) \quad$ для $i \in\{2, \ldots, d\}$.

Если $\mu_{\mathrm{A}}^{v} \in \mathrm{K}(X)$ и $b_{11}=d$, то $v_{1} \geq d-1$ согласно п. 1. Из каноничности метрики $\mu_{\mathrm{A}}^{v}$ следует, что $v_{1}=d-1$ и $v_{i}=d+i-2$ для $i \in\{2, \ldots, d\}$.

Пусть $\mu_{\mathrm{A}}^{v}-$ каноническая метрика, $\mu_{\mathrm{A}}^{v^{\prime}}-$ произвольная метрика. Покажем, что $v_{i} \leq v_{i}^{\prime}, i=1, \ldots, d$.

Очевидно, что для любых $\alpha, \alpha^{\prime}, \gamma \in X$ выполняются неравенства

$$
\begin{gathered}
\mu_{\mathrm{A}}^{v^{\prime}}\left(\alpha, \alpha^{\prime}\right) \leq \mu_{\mathrm{A}}^{v^{\prime}}(\alpha, \gamma)+\mu_{\mathrm{A}}^{v^{\prime}}\left(\gamma, \alpha^{\prime}\right), \mu_{\mathrm{A}}^{v^{\prime}}(\alpha, \gamma) \leq \mu_{\mathrm{A}}^{v^{\prime}}\left(\alpha, \alpha^{\prime}\right)+\mu_{\mathrm{A}}^{v^{\prime}}\left(\gamma, \alpha^{\prime}\right), \\
\mu_{\mathrm{A}}^{v^{\prime}}(\alpha, \gamma) \leq \mu_{\mathrm{A}}^{v^{\prime}}\left(\alpha, \alpha^{\prime}\right)+\mu_{\mathrm{A}}^{v^{\prime}}\left(\gamma, \alpha^{\prime}\right) .
\end{gathered}
$$


Для $(\alpha, \beta) \in X^{2}$ положим

$$
\delta(\alpha, \beta)=\mu_{\mathrm{A}}^{v^{\prime}}(\alpha, \beta)-\mu_{\mathrm{A}}^{v}(\alpha, \beta) .
$$

Из (2.6) получаем, что для любых $\alpha, \alpha^{\prime}, \gamma \in X$ справедливы неравенства

$$
\begin{gathered}
\delta(\alpha, \gamma) \leq \delta\left(\alpha, \alpha^{\prime}\right)+\delta\left(\gamma, \alpha^{\prime}\right), \quad \delta(\alpha, \gamma) \leq \delta\left(\alpha, \alpha^{\prime}\right)+\delta\left(\gamma, \alpha^{\prime}\right), \\
\delta\left(\alpha, \alpha^{\prime}\right) \leq \delta(\alpha, \gamma)+\delta\left(\gamma, \alpha^{\prime}\right) .
\end{gathered}
$$

Если все неравенства $v_{i} \leq v_{i}^{\prime}, i=1, \ldots, d$, не выполняются, то найдётся пара $\left(\alpha, \alpha^{\prime}\right) \in X^{2}$, для которой $\delta\left(\alpha, \alpha^{\prime}\right)<0$. Тогда из соотношений (2.7) следует одновременная справедливость неравенств

$$
\delta(\alpha, \gamma)<\delta\left(\gamma, \alpha^{\prime}\right), \quad \delta\left(\gamma, \alpha^{\prime}\right)<\delta(\alpha, \gamma),
$$

что невозможно. Отсюда получаем $\delta\left(\alpha, \alpha^{\prime}\right) \geq 0$. Значит, выполняются неравенства $v_{i} \leq v_{i}^{\prime}, i=1, \ldots, d$.

Таким образом, для канонической метрики $\mu_{\mathrm{A}}^{v}$ выполняются неравенства

$$
v_{1}^{\prime} \leq d-1, v_{i}^{\prime} \leq 2(d-1), i \in\{2, \ldots, d\} .
$$

4. Из определения канонической метрики следует, что $\mathrm{K}_{v}(X) \cap$ $\cap \mathrm{K}_{v^{\prime}}(X)=\varnothing$ для всех $v \neq v^{\prime}, v, v^{\prime} \in \Upsilon$. Очевидно, что $\mathrm{K}_{v}(X) \subset \mathrm{K}(X)$ для всех $v \in \Upsilon$.

Докажем включение $\mathrm{K}(X) \subseteq \bigcup_{v \in \mathrm{Y}} \mathrm{K}_{v}(X)$. Предположим, что $\mu_{\mathrm{A}} \notin$ $\notin \bigcup_{v \in \mathrm{Y}} \mathrm{K}_{v}(X)$ для некоторой метрики $\mu_{\mathrm{A}} \in \mathrm{K}(X), \quad \mathrm{A}=\left(\bar{A}_{1}, \ldots, \bar{A}_{d}\right) \in \overline{\mathbf{X}}^{(2)}$, $2 \leq d \leq C_{|X|}^{2}$. Причём $\mu_{\mathrm{A}}=\mu_{\mathrm{A}}^{v}, \quad$ где $\quad v=\left(v_{1}, \ldots, v_{d}\right) \in \mathbb{N}^{d} \backslash \Upsilon_{d}$. Включение $\mu_{\mathrm{A}} \in \bigcup_{v \in \mathrm{Y}} \mathrm{K}_{v}(X)$ для каждой метрики $\mu_{\mathrm{A}} \in \mathrm{K}(X)$ следует из п. 3 данной леммы. Лемма доказана.

\section{§ 3. Подметрики натуральной метрики}

В работе [5] было введено понятие подметрики метрики Хемминга. Это понятие естественным образом обобщается на произвольные конечные целочисленные метрики. Подметрики конечных целочисленных метрик могут возникать при необходимости укрупнения метрической классификации различных комбинаторных объектов или, например, при реализации поэтапного декодирования. 
Пусть $\mu-$ конечная целочисленная метрика на множестве $X$. Метрика $\rho_{\mu}: X \times X \rightarrow \mathbb{N}_{0}$, удовлетворяющая для любых $\alpha, \beta, \gamma, \delta \in X$ условиям:

1) если $\mu(\alpha, \beta)=\mu(\gamma, \delta)$, то $\rho_{\mu}(\alpha, \beta)=\rho_{\mu}(\gamma, \delta)$,

2) $\rho_{\mu}(\alpha, \beta) \leq \mu(\alpha, \beta)$,

называется подметрикой метрики $\mu$.

В работах [5], [6] описаны групповые подметрики метрики Хемминга. В данном параграфе будут рассмотрены некоторые общие свойства подметрик произвольной натуральной метрики. Некоторые свойства подметрик произвольной конечной целочисленной метрики могут быть получены из свойств натуральной метрики.

Для исследования подметрик натуральной метрики необходимо описать свойства её метрических орбиталов. Приведём необходимые и достаточные условия, которым должны удовлетворять метрические орбиталы подметрик произвольной натуральной метрики.

Утверждение 3.1. Пусть $2 \leq r<d,\left(\bar{A}_{1}, \ldots, \bar{A}_{d}\right),\left(\bar{B}_{1}, \ldots, \bar{B}_{r}\right) \in \overline{\mathbf{X}}^{(2)}$, метрика $\mu: X \times X \rightarrow\{0, \ldots, d\}$ задана условиями

$$
\mu\left(\alpha, \alpha^{\prime}\right)=i, \text { если }\left(\alpha, \alpha^{\prime}\right) \in \bar{A}_{i}, i \in\{1, \ldots, d\} .
$$

Метрика $\rho: X \times X \rightarrow\{0, \ldots, r\}$, где

$$
\rho\left(\alpha, \alpha^{\prime}\right)=i, \text { если }\left(\alpha, \alpha^{\prime}\right) \in \bar{B}_{i}, i \in\{1, \ldots, r\},
$$

тогда и только тогда является подметрикой метрики $\mu$, когда существует такое нетривиальное разбиение $U_{1}, \ldots, U_{r}$ множества $\{1, \ldots, d\}$, что для каждого $i \in\{1, \ldots, r\}$ справедливы соотношения:

1) $\bar{B}_{i}=\bigcup_{j \in U_{i}} \bar{A}_{j}$,

2) $i \leq \min \left\{j \mid j \in U_{i}\right\}$.

Доказательство тривиально.

Следующее утверждение позволяет рекурсивно перечислить все натуральные подметрики данной натуральной метрики $\mu$.

Утверждение 3.2. Пусть $2 \leq r<d,\left(\bar{A}_{1}, \ldots, \bar{A}_{d}\right),\left(\bar{B}_{1}, \ldots, \bar{B}_{r}\right) \in \overline{\mathbf{X}}^{(2)}$, метрика $\mu: X \times X \rightarrow\{0, \ldots, d\}$ задана условиями

$$
\mu\left(\alpha, \alpha^{\prime}\right)=i, \text { если }\left(\alpha, \alpha^{\prime}\right) \in \bar{A}_{i}, i \in\{1, \ldots, d\},
$$


и $U_{1}, \ldots, U_{r}-$ такое разбиение множества $\{1, \ldots, d\}$, что $i \leq \min \left\{j \mid j \in U_{i}\right\}$ для каждого $i \in\{1, \ldots, r\}$. Пусть также $\bar{B}_{i}=\bigcup_{j \in U_{i}} \bar{A}_{j}, \bar{H}_{i}=I \cup \bigcup_{j=1}^{i} \bar{B}_{j}, i \in\{1, \ldots, r\}$. Тогда функиия $\rho_{\mu}: X \times X \rightarrow \mathbb{N}_{0}$,

$$
\rho_{\mu}\left(\alpha, \alpha^{\prime}\right)=i, \text { если }\left(\alpha, \alpha^{\prime}\right) \in \bar{B}_{i}, i \in\{1, \ldots, r\},
$$

является натуральной подметрикой метрики $\mu$, если для всех $t \in\{2, \ldots, r\}$ справедливо включение

$$
\bigcup_{j=1}^{t-1}\left(\bigcup_{i_{j} \in U_{j}, i_{t-j} \in U_{t-j}} \bar{A}_{i_{j}} \circ \bar{A}_{i_{t-j}}\right) \subseteq \bar{H}_{t} .
$$

Доказательство следует из утверждений 2.1 и 3.1 .

Из утверждения 3.2 следует, что описание всех $(r+1)$-значных подметрик заданной $(d+1)$-значной метрики $\mu$,

$$
\mu\left(\alpha, \alpha^{\prime}\right)=i, \text { если }\left(\alpha, \alpha^{\prime}\right) \in \bar{A}_{i}, i \in\{1, \ldots, d\},
$$

сводится к нахождению всех таких $r$-разбиений $U_{1}, \ldots, U_{r}$ множества $\{1, \ldots, d\}$, что:

1) $t \leq \min \left\{j \mid j \in U_{t}\right\}$ для $t=\{1, \ldots, r\}$;

2) $\bigcup_{j=1}^{t-1}\left(\bigcup_{i_{j} \in U_{j}, i_{t-j} \in U_{t-j}} \bar{A}_{i_{j}} \circ \bar{A}_{i_{t-j}}\right) \subseteq \bar{H}_{t}$ для всех $t \in\{2, \ldots, r\}$,

где множество $\bar{H}_{i}=I \cup \bigcup_{j=1}^{i} \bar{B}_{j}$ определено в утверждении 3.2 .

Число $\delta(d, r)$ всех $r$-разбиений множества $\{1, \ldots, d\}, d \geq 2$, соответствующих $(r+1)$-значным подметрикам, меньше числа Стирлинга 2-го рода $\sigma(d, r)$ и удовлетворяет рекуррентному соотношению

$$
\delta(d, r)=\sum_{k=1}^{r} \delta(r, k) \sum_{l=0}^{d-r} C_{d-r}^{l} k^{l} \sigma(d-r-l, r-k),
$$

где $\sigma(t, 1)=\sigma(t, t)=1$ для любого натурального $t$.

Каждая метрика содержит в качестве подметрики тривиальную (двухзначную) метрику. Из других подметрик натуральной $(d+1)$-значной метрики проще всего описать её натуральные 3-значные и $d$-значные подметрики.

Утверждение 3.3. Пусть $d \geq 2,\left(\bar{A}_{1}, \ldots, \bar{A}_{d}\right) \in \overline{\mathbf{X}}^{(2)}, \mu: X \times X \rightarrow\{0, \ldots, d\}$,

$$
\mu\left(\alpha, \alpha^{\prime}\right)=i, \text { если }\left(\alpha, \alpha^{\prime}\right) \in \bar{A}_{i}, i \in\{1, \ldots, d\},
$$


является произвольной $(d+1)$-значной метрикой, $\bar{H}_{t}=\bigcup_{c=1}^{t} \bar{A}_{c} \cup I, t \in\{1, \ldots, d\}$. Tогда:

1) любое 2-разбиение $U_{1}, U_{2}$, где $1 \in U_{1}$, множества $\{1, \ldots, d\}$ задает 3-значную натуральную подметрику

$$
\rho_{\mu}\left(\alpha, \alpha^{\prime}\right)=\left\{\begin{array}{l}
1, \text { если }\left(\alpha, \alpha^{\prime}\right) \in \bigcup_{i \in U_{1}} \bar{A}_{i}, \\
2-\text { в остальных случаях }
\end{array}\right.
$$

метрики $\mu$; число 3-значных натуральных подметрик метрики $\mu$ равно $2^{d-1}-1$;

2) для $i, j \in\{1, \ldots, d\}, i<j$, функиия $\rho_{\mu}^{(i, j)}: X \times X \rightarrow \mathbb{N}_{0}$,

$$
\rho_{\mu}^{(i, j)}\left(\alpha, \alpha^{\prime}\right)= \begin{cases}i, & \text { если }\left(\alpha, \alpha^{\prime}\right) \in \bar{A}_{i} \cup \bar{A}_{j}, \\ t-1, & \text { если }\left(\alpha, \alpha^{\prime}\right) \in \bar{A}_{t}, t>j, \\ t, & \text { если }\left(\alpha, \alpha^{\prime}\right) \in \bar{A}_{t}, t<j, t \neq i .\end{cases}
$$

является $d$-значной натуральной метрикой, если для каждого $t, i<t<d$, справедливы включения:
a) $\left(\bar{A}_{j} * \bar{A}_{t-i}\right) \backslash \bar{H}_{t-1} \subseteq \bar{A}_{t} \cup \bar{A}_{j}$ npu $i<t<j, \quad t \neq 2 i$;
б) $\left(\left(\bar{A}_{j} * \bar{A}_{t-i}\right) \cup\left(\bar{A}_{j} \circ \bar{A}_{j}\right)\right) \backslash \bar{H}_{t-1} \subseteq \bar{A}_{t} \cup \bar{A}_{j}$ npu $i<t<j, \quad t=2 i$;
в) $\left(\bar{A}_{j} * \bar{A}_{t-i}\right) \backslash H_{t} \subseteq \bar{A}_{t+1}$ npu $t>j, t<i+j, t \neq 2 i$;
г) $\left(\bar{A}_{j} * \bar{A}_{t-i+1}\right) \backslash \bar{H}_{t} \subseteq \bar{A}_{t+1}$ npu $t>j, t \geq i+j$;
д) $\left(\left(\bar{A}_{j} * \bar{A}_{i}\right) \cup\left(\bar{A}_{j} \circ \bar{A}_{j}\right)\right) \backslash H_{t} \subseteq \bar{A}_{t+1}$ npu $t>j, j \neq i+1, \quad t=2 i$.

\section{Доказательство.}

1. Из утверждения 3.1 следует, что $\rho_{\mu}$ - подметрика. Число таких 2-разбиений $U_{1}, U_{2}$ множества $\{1, \ldots, d\}$, что $1 \in U_{1},\left|U_{1}\right|=k,\left|U_{2}\right|=d-k$, равно $C_{d-1}^{k-1}$. Число всех разбиений с $1 \in U_{1}$ равно

$$
\sum_{k=1}^{d-1} C_{d-1}^{k-1}=2^{d-1}-1
$$

2. Доказательство п. 2 имеет технический характер, и мы его не приводим. 2011, T. 2, № 4, С. 49-74 
Для иллюстрации применения утверждения 3.3 приведём классификацию всех подметрик произвольных натуральных 5- и 6-значных метрик.

Следствие 3.4. Пусть $\mu: X \times X \rightarrow \mathbb{N}_{0}$,

$$
\mu\left(\alpha, \alpha^{\prime}\right)=i, \text { если }\left(\alpha, \alpha^{\prime}\right) \in \bar{A}_{i}, i \in\{1, \ldots, 4\},
$$

является произвольной натуральной 5-значной метрикой. Тогда 4-значные подметрики метрики $\mu$ имеют вид:

1) если $\left(\bar{A}_{2} \circ \bar{A}_{2}\right) \backslash H_{1} \subseteq \bar{A}_{3}$, то

$$
\rho_{\mu}^{(1,2)}\left(\alpha, \alpha^{\prime}\right)=\left\{\begin{array}{l}
1, \quad \text { если }\left(\alpha, \alpha^{\prime}\right) \in \bar{A}_{1} \cup \bar{A}_{2}, \\
i-1, \text { если }\left(\alpha, \alpha^{\prime}\right) \in \bar{A}_{i}, i \in\{3,4\} ;
\end{array}\right.
$$

2) если $\left(\bar{A}_{1} * \bar{A}_{3} \cup \bar{A}_{3} \circ \bar{A}_{3}\right) \backslash \bar{H}_{1} \subseteq \bar{A}_{2}$, то

$$
\rho_{\mu}^{(1,3)}\left(\alpha, \alpha^{\prime}\right)=\left\{\begin{array}{l}
1, \text { если }\left(\alpha, \alpha^{\prime}\right) \in \bar{A}_{1} \cup \bar{A}_{3}, \\
2, \text { если }\left(\alpha, \alpha^{\prime}\right) \in \bar{A}_{2}, \\
3, \text { если }\left(\alpha, \alpha^{\prime}\right) \in \bar{A}_{4} ;
\end{array}\right.
$$

3) если $\left(\bar{A}_{1} * \bar{A}_{4} \cup \bar{A}_{4} \circ \bar{A}_{4}\right) \backslash \bar{H}_{1} \subseteq \bar{A}_{2}$, то

$$
\rho_{\mu}^{(1,4)}\left(\alpha, \alpha^{\prime}\right)=\left\{\begin{array}{l}
1, \text { если }\left(\alpha, \alpha^{\prime}\right) \in \bar{A}_{1} \cup \bar{A}_{4}, \\
i, \text { если }\left(\alpha, \alpha^{\prime}\right) \in \bar{A}_{i}, i \in\{2,3\} ;
\end{array}\right.
$$

4) для любых множеств $\bar{A}_{1}, \bar{A}_{2}, \bar{A}_{3}, \bar{A}_{4}$, задающих метрику $\mu$,

$$
\begin{gathered}
\rho_{\mu}^{(2,4)}\left(\alpha, \alpha^{\prime}\right)=\left\{\begin{array}{l}
2, \text { если }\left(\alpha, \alpha^{\prime}\right) \in \bar{A}_{2} \cup \bar{A}_{4}, \\
i, \text { если }\left(\alpha, \alpha^{\prime}\right) \in \bar{A}_{i}, i \in\{1,3\} ;
\end{array}\right. \\
\rho_{\mu}^{(2,3)}\left(\alpha, \alpha^{\prime}\right)=\left\{\begin{array}{l}
1, \text { если }\left(\alpha, \alpha^{\prime}\right) \in \bar{A}_{1}, \\
2, \text { если }\left(\alpha, \alpha^{\prime}\right) \in \bar{A}_{2} \cup \bar{A}_{3}, \\
3, \text { если }\left(\alpha, \alpha^{\prime}\right) \in \bar{A}_{4} ;
\end{array}\right. \\
\rho_{\mu}^{(3,4)}\left(\alpha, \alpha^{\prime}\right)=\left\{\begin{array}{l}
i, \text { если }\left(\alpha, \alpha^{\prime}\right) \in \bar{A}_{i}, i \in\{1,2\}, \\
3, \text { если }\left(\alpha, \alpha^{\prime}\right) \in \bar{A}_{3} \cup \bar{A}_{4} .
\end{array}\right.
\end{gathered}
$$

Доказательство непосредственно следует из утверждения 3.3.

Опишем теперь все подметрики произвольной натуральной 6-значной метрики. В утверждении 3.3 описаны 5- и 3-значные натуральные подмет- 
рики 6-значной метрики, осталось описать её 4-значные натуральные подметрики.

Следствие 3.5. Пусть $\mu: X \times X \rightarrow \mathbb{N}_{0}$,

$$
\mu\left(\alpha, \alpha^{\prime}\right)=i, \text { если }\left(\alpha, \alpha^{\prime}\right) \in \bar{A}_{i}, i \in\{1, \ldots, 5\},
$$

является произвольной 6-значной натуральной метрикой. Пусть также $\{i, j, k\}=\{3,4,5\}$. Тогда 4-значные натуральные подметрики метрики $\mu$ равны:

1) если $\bar{A}_{1} * \bar{A}_{i} \subseteq \bar{A}_{1} \cup \bar{A}_{2} \cup \bar{A}_{j} \cup \bar{A}_{i}$, mо

$$
\rho_{\mu, i, j}^{(1)}\left(\alpha, \alpha^{\prime}\right)=\left\{\begin{array}{l}
1, \text { если }\left(\alpha, \alpha^{\prime}\right) \in \bar{A}_{1} \cup \bar{A}_{i}, \\
2, \text { если }\left(\alpha, \alpha^{\prime}\right) \in \bar{A}_{2} \cup \bar{A}_{j}, \\
3, \text { если }\left(\alpha, \alpha^{\prime}\right) \in \bar{A}_{k} ;
\end{array}\right.
$$

2) если $A_{1} * A_{2} \cup A_{2} \circ A_{2} \subseteq I \cup \bar{A}_{1} \cup \bar{A}_{2} \cup \bar{A}_{j} \cup \bar{A}_{i}$, mо

$$
\rho_{\mu, i, j}^{(2)}\left(\alpha, \alpha^{\prime}\right)=\left\{\begin{array}{l}
1, \text { если }\left(\alpha, \alpha^{\prime}\right) \in \bar{A}_{1} \cup \bar{A}_{2}, \\
2, \text { если }\left(\alpha, \alpha^{\prime}\right) \in \bar{A}_{i} \cup \bar{A}_{j}, \\
3, \text { если }\left(\alpha, \alpha^{\prime}\right) \in \bar{A}_{k}
\end{array}\right.
$$

3) если $\bar{A}_{1} * \bar{A}_{k} \cup \bar{A}_{k} \circ \bar{A}_{k} \subseteq I \cup \bar{A}_{1} \cup \bar{A}_{2} \cup \bar{A}_{k}$, mо

$$
\rho_{\mu, i, j}^{(3)}\left(\alpha, \alpha^{\prime}\right)=\left\{\begin{array}{l}
1, \text { если }\left(\alpha, \alpha^{\prime}\right) \in \bar{A}_{1} \cup \bar{A}_{k}, \\
2, \text { если }\left(\alpha, \alpha^{\prime}\right) \in \bar{A}_{2}, \\
3, \text { если }\left(\alpha, \alpha^{\prime}\right) \in \bar{A}_{j} \cup \bar{A}_{i} ;
\end{array}\right.
$$

4) для произвольных множеств $\bar{A}_{1}, \bar{A}_{2}, \bar{A}_{3}$, задающчих метрику $\mu$,

$$
\begin{gathered}
\rho_{\mu, i, j}^{(4)}\left(\alpha, \alpha^{\prime}\right)=\left\{\begin{array}{l}
1, \text { если }\left(\alpha, \alpha^{\prime}\right) \in \bar{A}_{1}, \\
2, \text { если }\left(\alpha, \alpha^{\prime}\right) \in \bar{A}_{2} \cup \bar{A}_{k}, \\
3, \text { если }\left(\alpha, \alpha^{\prime}\right) \in \bar{A}_{j} \cup \bar{A}_{i} ;
\end{array}\right. \\
\rho_{\mu}^{(5)}\left(\alpha, \alpha^{\prime}\right)=\left\{\begin{array}{l}
1, \text { если }\left(\alpha, \alpha^{\prime}\right) \in \bar{A}_{1}, \\
2, \text { если }\left(\alpha, \alpha^{\prime}\right) \in \bar{A}_{2}, \\
3, \text { если }\left(\alpha, \alpha^{\prime}\right) \in \bar{A}_{j} \cup \bar{A}_{i} \cup \bar{A}_{k} ;
\end{array}\right.
\end{gathered}
$$




$$
\rho_{\mu, i, j}^{(6)}\left(\alpha, \alpha^{\prime}\right)=\left\{\begin{array}{l}
1, \text { если }\left(\alpha, \alpha^{\prime}\right) \in \bar{A}_{1}, \\
2, \text { если }\left(\alpha, \alpha^{\prime}\right) \in \bar{A}_{j} \cup \bar{A}_{i} \cup \bar{A}_{2}, \\
3, \text { если }\left(\alpha, \alpha^{\prime}\right) \in \bar{A}_{k}
\end{array}\right.
$$

5) если $\left(\bar{A}_{1} \cup \bar{A}_{2} \cup \bar{A}_{i}\right) \circ\left(\bar{A}_{1} \cup \bar{A}_{2} \cup \bar{A}_{i}\right) \subseteq I \cup \bar{A}_{1} \cup \bar{A}_{2} \cup \bar{A}_{i} \cup \bar{A}_{j}$, mo

$$
\rho_{\mu, i, j}^{(7)}\left(\alpha, \alpha^{\prime}\right)=\left\{\begin{array}{l}
1, \text { если }\left(\alpha, \alpha^{\prime}\right) \in \bar{A}_{1} \cup \bar{A}_{2} \cup \bar{A}_{i}, \\
2, \text { если }\left(\alpha, \alpha^{\prime}\right) \in \bar{A}_{j}, \\
3, \text { если }\left(\alpha, \alpha^{\prime}\right) \in \bar{A}_{k} ;
\end{array}\right.
$$

6) если $\left(\bar{A}_{1} \cup \bar{A}_{i} \cup \bar{A}_{j}\right) \circ\left(\bar{A}_{1} \cup \bar{A}_{i} \cup \bar{A}_{j}\right) \subseteq I \cup \bar{A}_{1} \cup \bar{A}_{2} \cup \bar{A}_{i} \cup \bar{A}_{j}$, mo

$$
\rho_{\mu, i, j}^{(8)}\left(\alpha, \alpha^{\prime}\right)=\left\{\begin{array}{l}
1, \text { если }\left(\alpha, \alpha^{\prime}\right) \in \bar{A}_{1} \cup \bar{A}_{i} \cup \bar{A}_{j}, \\
2, \text { если }\left(\alpha, \alpha^{\prime}\right) \in \bar{A}_{2}, \\
3, \text { если }\left(\alpha, \alpha^{\prime}\right) \in \bar{A}_{k} .
\end{array}\right.
$$

Доказательство следует из следствия 1.2 и утверждения 3.2.

Ясно, что все подметрики, приведенные в следствиях 3.4 и 3.5, являются натуральными.

\section{§ 4. Надметрики натуральной метрики}

В данном параграфе вводится понятие надметрики конечной целочисленной метрики и рассматриваются общие свойства надметрик натуральных метрик. Надметрики могут использоваться для более тонкой классификации комбинаторных объектов, чем при использовании самой метрики.

Пусть $\mu$ - конечная целочисленная метрика на множестве $X$. Метрика $\rho_{\mu}: X \times X \rightarrow \mathbb{N}_{0}$ называется надметрикой метрики $\mu$, если для любых $\alpha, \beta, \gamma, \delta \in X$ она удовлетворяет условиям:

1) если $\rho_{\mu}(\alpha, \beta)=\rho_{\mu}(\gamma, \delta)$, то $\mu(\alpha, \beta)=\mu(\gamma, \delta)$,

2) $\quad \mu(\alpha, \beta) \leq \rho_{\mu}(\alpha, \beta)$.

Очевидно, что метрика $\mu$ является подметрикой метрики $\rho_{\mu}$.

Для исследования надметрик натуральной метрики необходимо описать свойства её метрических орбиталов. Приведём необходимые и достаточные условия, которым должны удовлетворять метрические орбиталы надметрик произвольной натуральной метрики. 
Утверждение 4.1. Пусть $2 \leq d<r,\left(\bar{A}_{1}, \ldots, \bar{A}_{d}\right),\left(\bar{B}_{1}, \ldots, \bar{B}_{r}\right) \in \overline{\mathbf{X}}^{(2)}$ и натуральная метрика $\mu: X \times X \rightarrow\{0, \ldots, d\}$ задана условиями

$$
\mu\left(\alpha, \alpha^{\prime}\right)=i, \text { если }\left(\alpha, \alpha^{\prime}\right) \in \bar{A}_{i}, i \in\{1, \ldots, d\} .
$$

Натуральная метрика $\rho: X \times X \rightarrow\{0, \ldots, r\}$, заданная условиями

$$
\rho_{\mu}^{(i, j)}\left(\alpha, \alpha^{\prime}\right)=i, \text { если }\left(\alpha, \alpha^{\prime}\right) \in \bar{B}_{i}, i \in\{1, \ldots, r\}
$$

тогда и только тогда является надметрикой метрики $\mu$, когда существует такое разбиение $U_{1}, \ldots, U_{d}$ множества $\{1, \ldots, r\}$, что для каждого $i \in\{1, \ldots, d\}$ справедливы соотношения:

1)

$$
\begin{aligned}
& \text { 1) } \bar{A}_{i}=\bigcup_{j \in U_{i}} \bar{B}_{j}, \\
& \text { 2) } i \leq \min \left\{j \mid j \in U_{i}\right\} .
\end{aligned}
$$

\section{Доказательство тривиально.}

В отличие от описания подметрик данной метрики аналогичная задача для надметрик является более трудной. Это связано, в частности, с бесконечностью множества целочисленных надметрик. Для иллюстрации этого положения приведём описание $(d+2)$-значных натуральных надметрик $(d+1)$-значной натуральной метрики.

Утверждение 4.2. Пусть $d \geq 2,\left(\bar{A}_{1}, \ldots, \bar{A}_{d}\right) \in \overline{\mathbf{X}}^{(2)}, \mu: X \times X \rightarrow \mathbb{N}_{0}$,

$$
\mu\left(\alpha, \alpha^{\prime}\right)=i, \text { если }\left(\alpha, \alpha^{\prime}\right) \in \bar{A}_{i}, i \in\{1, \ldots, d\}
$$

является $(d+1)$-значной метрикой, $\bar{H}_{t}=\bigcup_{c=1}^{t} \bar{A}_{c} \cup I, t \in\{1, \ldots, d\}$. Пусть числа $i \in\{1, \ldots, d\}, \quad j \in\{1, \ldots, d+1\}$ и разбиение $\bar{A}_{i}^{\prime}, \bar{A}_{i}^{\prime \prime}$ множества $\bar{A}_{i}-$ произвольное фиксированное. Функция $\rho_{\mu, A_{i}^{\prime}}^{(i, j)}: X \times X \rightarrow \mathbb{N}_{0}$,

$$
\rho_{\mu, A_{i}^{\prime}}^{(i, j)}\left(\alpha, \alpha^{\prime}\right)=\left\{\begin{array}{l}
t, \text { если }\left(\alpha, \alpha^{\prime}\right) \in \bar{A}_{t}, t \in\{1, \ldots, j-1\} \backslash\{i\}, \\
i, \text { если }\left(\alpha, \alpha^{\prime}\right) \in \bar{A}_{i}^{\prime}, \\
j, \text { если }\left(\alpha, \alpha^{\prime}\right) \in \bar{A}_{i}^{\prime \prime}, \\
t+1, \text { если }\left(\alpha, \alpha^{\prime}\right) \in \bar{A}_{t}, t \in\{j, \ldots, d\},
\end{array}\right.
$$


является $(d+2)$-значной метрикой тогда и только тогда, когда $i<j$ и для каждого $t \in\{1, \ldots, d\}$ справедливы соотночения

$$
\begin{aligned}
& \left(\bigcup_{c=1}^{i-1}\left(\bar{A}_{c} \circ \bar{A}_{i-c}\right)\right) \backslash \bar{H}_{i-1} \subseteq \bar{A}_{i}^{\prime}, \\
& \left(\bar{A}_{i}^{\prime} * \bar{A}_{t-i}\right) \cup \bigcup_{c \in\{1, \ldots, t-1\} \backslash\{i, t-i\}}\left(\bar{A}_{c} \circ \bar{A}_{t-c}\right) \subseteq \begin{cases}\left(\bar{H}_{t} \backslash \bar{A}_{i}^{\prime \prime}\right), & \text { если } t \in\{1, \ldots, j-1\} \backslash\{i, 2 i\}, \\
\bar{H}_{t}, & \text { если } t=j, j \neq 2 i,\end{cases} \\
& \left(\bar{A}_{i}^{\prime} \circ \bar{A}_{i}^{\prime}\right) \cup \bigcup_{c \in\{1, \ldots, t-1\} \backslash i\}}\left(\bar{A}_{c} \circ \bar{A}_{t-c}\right) \subseteq \begin{cases}\bar{H}_{t} \backslash \bar{A}_{i}^{\prime \prime}, & \text { если } t=2 i, t<j, \\
\bar{H}_{t}, & \text { если } t=j=2 i,\end{cases} \\
& \left(\bar{B}_{t-j}^{(i, j)} * \bar{A}_{i}\right) \cup \bigcup_{c \in\{1, \ldots, t-1\} \backslash\{i, t-i, j, t-j\}}\left(\bar{B}_{c}^{(i, j)} \circ \bar{B}_{t-c}^{(i, j)}\right) \subseteq \bar{H}_{t-1} \text { при } t \notin\{2 i, j+i, 2 j\}, \\
& \left(\bar{A}_{i}^{\prime \prime} * \bar{B}_{t-j}^{(i, j)}\right) \bigcup\left(\bar{A}_{i}^{\prime} \circ \bar{A}_{i}^{\prime}\right) \bigcup \bigcup_{c \in\{1, \ldots, t-1\} \backslash\{i, j, t-j\}}\left(\bar{B}_{c}^{(i, j)} \circ \bar{B}_{t-c}^{(i, j)}\right) \subseteq \bar{H}_{t-1} \text { при } t=2 i, \\
& \left(\bar{A}_{i}^{\prime} * \bar{B}_{t-j}^{(i, j)}\right) \cup\left(\bar{A}_{i}^{\prime \prime} \circ \bar{A}_{i}^{\prime \prime}\right) \bigcup \bigcup_{c \in\{1, \ldots, t-1\} \backslash\{i, j, t-j\}}\left(\bar{B}_{c}^{(i, j)} \circ \bar{B}_{t-c}^{(i, j)}\right) \subseteq \bar{H}_{t-1} \text { при } t=2 j, \\
& \left(\bar{A}_{i}^{\prime} * \bar{A}_{i}^{\prime \prime}\right) \cup \bigcup_{c \in\{1, \ldots, t-1\} \backslash\{i, j\}}\left(\bar{B}_{c}^{(i, j)} \circ \bar{B}_{t-c}^{(i, j)}\right) \subseteq \bar{H}_{t-1} \text { при } t=i+j,
\end{aligned}
$$

где

$$
\bar{B}_{c}^{(i, j)}=\left\{\begin{array}{l}
\bar{A}_{c}, \text { если } c \in\{1, \ldots, t-1\} \backslash\{i\}, \\
\bar{A}_{c-1}, \text { если } c \in\{j, \ldots, d\} .
\end{array}\right.
$$

Доказательство. Обозначим

$$
\bar{B}_{t}=\left\{\left(\alpha, \alpha^{\prime}\right) \in X \times X \mid \rho_{\mu, A_{i}^{\prime}}^{(i, j)}\left(\alpha, \alpha^{\prime}\right)=t\right\}, t=1, \ldots, d+1 .
$$

Условие $i<j$ получается из утверждения 4.1. Из теоремы 2.1 следует, что для каждого $t \in\{1, \ldots, d\}$ справедливо включение

$$
\bigcup_{c=1}^{t-1} \bar{A}_{c} \circ \bar{A}_{t-c} \subseteq \bar{H}_{t}
$$

Также из следствия 2.2 получаем, что функция $\rho_{\mu, A_{i}^{\prime}}^{(i, j)}-$ метрика, если для каждого $t \in\{1, \ldots, d\}$ справедливо включение

$$
\bigcup_{c=1}^{t-1}\left(\bar{B}_{c} \circ \bar{B}_{t-c}\right) \subseteq \bar{H}_{t}^{\prime}, \quad \text { где } \bar{H}_{t}^{\prime}=I \bigcup \bigcup_{c=1}^{t} \bar{B}_{c} .
$$


Если $t<i$, то $\bar{B}_{c}=\bar{A}_{c}$ для всех $c \in\{1, \ldots, i-1\}$ и включение (4.2) равносильно включению (4.1). Если $t=i$, то включение (4.2) равносильно соотношению

$$
\bigcup_{c=1}^{i-1}\left(\bar{A}_{c} \circ \bar{A}_{t-c}\right) \backslash \bar{H}_{t-1} \subseteq \bar{A}_{i}^{\prime} .
$$

Если же $i<t<j$, то $\bar{B}_{c}=\bar{A}_{c}$ для всех $c \in\{i+1, \ldots, j-1\}$ и включение (4.2) принимает вид

$$
\begin{gathered}
\left(\bar{A}_{i}^{\prime} * \bar{A}_{t-i}\right) \cup \bigcup_{c \in\{1, \ldots, t-1\} \backslash\{, t-i\}}\left(\bar{A}_{c} \circ \bar{A}_{t-c}\right) \subseteq I \cup \bar{A}_{i}^{\prime} \cup \bigcup_{c \in\{1, \ldots, t\} \backslash\{i\}} \bar{A}_{c} \text { при } t \neq 2 i, \\
\bar{A}_{i}^{\prime} \circ \bar{A}_{i}^{\prime} \cup \bigcup_{c \in\{1, \ldots, t-1\} \backslash\{i\}}\left(\bar{A}_{c} \circ \bar{A}_{t-c}\right) \subseteq I \cup \bar{A}_{i}^{\prime} \cup \bigcup_{c \in\{1, \ldots, t\} \backslash\{i\}} \bar{A}_{c} \text { при } t=2 i .
\end{gathered}
$$

Если $t=j$, то должно выполняться соотношение

$$
\begin{gathered}
\left(\bar{A}_{i}^{\prime} * \bar{A}_{t-i}\right) \cup \bigcup_{c \in\{1, \ldots, j-1\} \backslash\{i, j-i\}}\left(\bar{A}_{c} \circ \bar{A}_{t-c}\right) \subseteq I \cup \bar{A}_{i}^{\prime} \cup \bar{A}_{i}^{\prime \prime} \cup \bigcup_{c \in\{1, \ldots, j-1\} \backslash\{i\}} \bar{A}_{c} \text { при } t \neq 2 i, \\
\left(\bar{A}_{i}^{\prime} \circ \bar{A}_{i}^{\prime}\right) \cup \bigcup_{c \in\{1, \ldots, t-1\} \backslash\{i\}}\left(\bar{A}_{c} \circ \bar{A}_{t-c}\right) \subseteq I \cup \bar{A}_{i}^{\prime} \cup \bar{A}_{i}^{\prime \prime} \cup \bigcup_{c \in\{1, \ldots, j-1\} \backslash\{i\}} \bar{A}_{c} \text { при } t=2 i,
\end{gathered}
$$

где

$$
I \cup \bar{A}_{i}^{\prime} \cup \bar{A}_{i}^{\prime \prime} \cup \bigcup_{c \in\{1, \ldots, j-1\} \backslash i\}} \bar{A}_{c}=\bar{H}_{j-1} .
$$

Если $j<t$, то $\bar{B}_{c}=\bar{A}_{c-1}$ для всех $c>j$, и включение (4.2) принимает вид

$$
\left(\bar{A}_{i}^{\prime \prime *} \bar{B}_{t-j}^{(i, j)}\right) \cup\left(\bar{A}_{i}^{\prime} * \bar{B}_{t-i}^{(i, j)}\right) \cup \bigcup_{c \in\{1, \ldots, t-1\} \backslash\{i, t i, j, t-j\}}\left(\bar{B}_{c}^{(i, j)} \circ \bar{B}_{t-c}^{(i, j)}\right) \subseteq \bar{H}_{t-1}
$$

при $t \notin\{2 i, j+i, 2 j\}$,

$$
\begin{aligned}
& \left(\bar{A}_{i}^{\prime \prime *} \bar{B}_{t-j}^{(i, j)}\right) \cup \bar{A}_{i}^{\prime} \circ \bar{A}_{i}^{\prime} \bigcup \bigcup_{c \in\{1, \ldots, t-1\} \backslash\{i, j, t-j\}}\left(\bar{B}_{c}^{(i, j)} \circ \bar{B}_{t-c}^{(i, j)}\right) \subseteq \bar{H}_{t-1} \text { при } t=2 i, \\
& \left(\bar{A}_{i}^{\prime} * \bar{B}_{t-j}^{(i, j)}\right) \cup\left(\bar{A}_{i}^{\prime \prime} \circ \bar{A}_{i}^{\prime \prime}\right) \cup \bigcup_{c \in\{1, \ldots, t-1\} \backslash\{i, j, t-j\}}\left(\bar{B}_{c}^{(i, j)} \circ \bar{B}_{t-c}^{(i, j)}\right) \subseteq \bar{H}_{t-1} \text { при } t=2 j, \\
& \left(\bar{A}_{i}^{\prime} * \bar{A}_{i}^{\prime \prime}\right) \cup \bigcup_{c \in\{1, \ldots, t-1\} \backslash\{i, j\}}\left(\bar{B}_{c}^{(i, j)} \circ \bar{B}_{t-c}^{(i, j)}\right) \subseteq \bar{H}_{t-1} \text { при } t=i+j .
\end{aligned}
$$

Утверждение доказано.

Утверждение 4.2 естественно применить для классификации натуральных надметрик 3-значной и 4-значных натуральных метрик. При этом, в частности, получается полное описание 5-значных метрик. Этот процесс можно использовать и для классификации метрик большей значности. 
Следствие 4.3. Пусть $\left(\bar{A}_{1}, \bar{A}_{2}\right) \in \overline{\mathbf{X}}^{(2)}$, функция $\mu: X \times X \rightarrow \mathbb{N}_{0}$, заданная условиями

$$
\mu\left(\alpha, \alpha^{\prime}\right)=i, \text { если }\left(\alpha, \alpha^{\prime}\right) \in \bar{A}_{i}, i=1,2,
$$

является произвольной 3-значной натуральной метрикой. Тогда её 4-значные натуральные надметрики, задающиеся разбиением $\bar{A}_{i}^{\prime}, \bar{A}_{i}^{\prime \prime}$ множества $\bar{A}_{i}, i \in\{1,2\}$, имеют следуюший вид:

1) если $\bar{A}_{1}^{\prime} \circ \bar{A}_{1}^{\prime} \subseteq \bar{A}_{1} \cup I$, mо

$$
\rho_{\mu, A_{1}^{\prime}}^{(1,2)}\left(\alpha, \alpha^{\prime}\right)=\left\{\begin{array}{l}
1, \text { если }\left(\alpha, \alpha^{\prime}\right) \in \bar{A}_{1}^{\prime}, \\
2, \text { если }\left(\alpha, \alpha^{\prime}\right) \in \bar{A}_{1}^{\prime \prime}, \\
3, \text { если }\left(\alpha, \alpha^{\prime}\right) \in \bar{A}_{2} ;
\end{array}\right.
$$

2) если $\bar{A}_{1}^{\prime} \circ \bar{A}_{1}^{\prime} \subseteq \bar{A}_{2} \cup \bar{A}_{1}^{\prime} \cup I$, то

$$
\rho_{\mu, A_{1}^{\prime}}^{(1,3)}\left(\alpha, \alpha^{\prime}\right)=\left\{\begin{array}{l}
1, \text { если }\left(\alpha, \alpha^{\prime}\right) \in \overline{A_{1}^{\prime}}, \\
2, \text { если }\left(\alpha, \alpha^{\prime}\right) \in \bar{A}_{2}, \\
3, \text { если }\left(\alpha, \alpha^{\prime}\right) \in \bar{A}_{1}^{\prime \prime} ;
\end{array}\right.
$$

3) если $\bar{A}_{1} \circ \bar{A}_{1} \subseteq \bar{A}_{2}^{\prime} \cup \bar{A}_{1} \cup I$, то

$$
\rho_{\mu, A_{1}^{\prime}}^{(2,3)}\left(\alpha, \alpha^{\prime}\right)=\left\{\begin{array}{l}
1, \text { если }\left(\alpha, \alpha^{\prime}\right) \in \bar{A}_{1}, \\
2, \text { если }\left(\alpha, \alpha^{\prime}\right) \in \bar{A}_{2}^{\prime}, \\
3, \text { если }\left(\alpha, \alpha^{\prime}\right) \in \bar{A}_{2}^{\prime \prime} .
\end{array}\right.
$$

Доказательство следует из утверждения 4.2.

Следствие 4.4. Пусть $\left(\bar{A}_{1}, \bar{A}_{2}, \bar{A}_{3}\right) \in \overline{\mathbf{X}}^{(2)}$, функция $\mu: X \times X \rightarrow \mathbb{N}_{0}$, заданная условиями

$$
\mu\left(\alpha, \alpha^{\prime}\right)=i, \text { если }\left(\alpha, \alpha^{\prime}\right) \in \bar{A}_{i}, i=1,2,3,
$$

является произвольной 4-значной натуральной метрикой. Тогда её натуральные надметрики, задающиеся разбиениями $\bar{A}_{i}=\bar{A}_{i}^{\prime} \cup \bar{A}_{i}^{\prime \prime}, i=1,2,3$, определяются равенствами:

1) если $\bar{A}_{1}^{\prime} \circ \bar{A}_{1}^{\prime} \subseteq \bar{A}_{1} \cup I$, mo

$$
\rho_{\mu, A_{1}^{\prime}}^{(1,2)}\left(\alpha, \alpha^{\prime}\right)=\left\{\begin{array}{l}
1, \text { если }\left(\alpha, \alpha^{\prime}\right) \in \bar{A}_{1}^{\prime}, \\
2, \text { если }\left(\alpha, \alpha^{\prime}\right) \in \bar{A}_{1}^{\prime \prime}, \\
3, \text { если }\left(\alpha, \alpha^{\prime}\right) \in \bar{A}_{2}, \\
4, \text { если }\left(\alpha, \alpha^{\prime}\right) \in \bar{A}_{3} ;
\end{array}\right.
$$


2) если $\overline{A_{1}^{\prime}} \circ \overline{A_{1}^{\prime}} \subseteq \bar{A}_{2} \cup \bar{A}_{1}^{\prime} \cup I, A_{1}^{\prime} * A_{2} \subseteq I \cup \bar{A}_{2} \cup \bar{A}_{1}$, mо

$$
\rho_{\mu, A_{1}^{\prime}}^{(1,3)}\left(\alpha, \alpha^{\prime}\right)=\left\{\begin{array}{l}
1, \text { если }\left(\alpha, \alpha^{\prime}\right) \in \bar{A}_{1}^{\prime}, \\
2, \text { если }\left(\alpha, \alpha^{\prime}\right) \in \bar{A}_{2}, \\
3, \text { если }\left(\alpha, \alpha^{\prime}\right) \in \bar{A}_{1}^{\prime \prime}, \\
4, \text { если }\left(\alpha, \alpha^{\prime}\right) \in \bar{A}_{3} ;
\end{array}\right.
$$

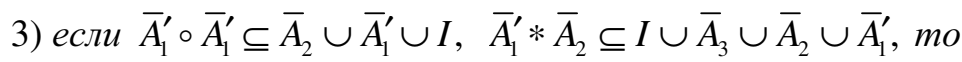

$$
\rho_{\mu, A_{1}^{\prime}}^{(1,4)}\left(\alpha, \alpha^{\prime}\right)=\left\{\begin{array}{l}
1, \text { если }\left(\alpha, \alpha^{\prime}\right) \in \bar{A}_{1}^{\prime}, \\
2, \text { если }\left(\alpha, \alpha^{\prime}\right) \in \bar{A}_{2}, \\
3, \text { если }\left(\alpha, \alpha^{\prime}\right) \in \bar{A}_{3}, \\
4, \text { если }\left(\alpha, \alpha^{\prime}\right) \in \bar{A}_{1}^{\prime \prime} ;
\end{array}\right.
$$

4) если $\overline{A_{1}} \circ \overline{A_{1}} \subseteq \overline{A_{2}^{\prime}} \cup \overline{A_{1}} \cup I, \bar{A}_{1} * \bar{A}_{2}^{\prime} \subseteq I \cup \bar{A}_{2} \cup \overline{A_{1}}$, mo

$$
\rho_{\mu, A_{1}^{\prime}}^{(2,3)}\left(\alpha, \alpha^{\prime}\right)=\left\{\begin{array}{l}
1, \text { если }\left(\alpha, \alpha^{\prime}\right) \in \bar{A}_{1}, \\
2, \text { если }\left(\alpha, \alpha^{\prime}\right) \in \bar{A}_{2}^{\prime}, \\
3, \text { если }\left(\alpha, \alpha^{\prime}\right) \in \bar{A}_{2}^{\prime \prime}, \\
4, \text { если }\left(\alpha, \alpha^{\prime}\right) \in \bar{A}_{3} ;
\end{array}\right.
$$

5) если $\bar{A}_{1} \circ \bar{A}_{1} \subseteq \bar{A}_{2}^{\prime} \cup \bar{A}_{1} \cup I, A_{1} * A_{2}^{\prime} \subseteq I \cup \bar{A}_{3} \cup \bar{A}_{2}^{\prime} \cup \bar{A}_{1}$, mo

$$
\rho_{\mu, A_{1}^{\prime}}^{(2,4)}\left(\alpha, \alpha^{\prime}\right)=\left\{\begin{array}{l}
1, \text { если }\left(\alpha, \alpha^{\prime}\right) \in \bar{A}_{1}, \\
2, \text { если }\left(\alpha, \alpha^{\prime}\right) \in \bar{A}_{2}^{\prime}, \\
3, \text { если }\left(\alpha, \alpha^{\prime}\right) \in \bar{A}_{3}, \\
4, \text { если }\left(\alpha, \alpha^{\prime}\right) \in \bar{A}_{2}^{\prime \prime} ;
\end{array}\right.
$$

6) если $A_{1} * A_{2} \subseteq I \cup \bar{A}_{3}^{\prime} \cup \bar{A}_{2} \cup \bar{A}_{1}$, то

$$
\rho_{\mu, A_{1}^{\prime}}^{(3,4)}\left(\alpha, \alpha^{\prime}\right)=\left\{\begin{array}{l}
1, \text { если }\left(\alpha, \alpha^{\prime}\right) \in \bar{A}_{1}, \\
2, \text { если }\left(\alpha, \alpha^{\prime}\right) \in \bar{A}_{2}, \\
3, \text { если }\left(\alpha, \alpha^{\prime}\right) \in \bar{A}_{3}^{\prime}, \\
4, \text { если }\left(\alpha, \alpha^{\prime}\right) \in \bar{A}_{3}^{\prime \prime} .
\end{array}\right.
$$

Доказательство проводится прямой проверкой соотношений утверждения 4.2. 
Б. А. Погорелов, М. А. Пудовкина

\section{Список литературы}

1. Габидулин Э. М. Теория кодов с максимальным ранговым расстоянием. - Проблемы передачи информации, 1985, т. 21, вып. 1, с. 1-12.

2. Кшевецкий A. С. Разработка новых кодов в ранговой метрике и криптосистем с открытым ключом. - МФТИ. Дисс. канд. физ.-мат. наук, 2007.

3. Левенштейн В. И. О совершенных кодах в метрике выпадений и вставок. - Дискретная математика, 1991, т. 3, вып. 1, с. 1-20.

4. Музычук M. Е. Подсхемы схемы Хэмминга. - Исследов. по алгебр. теории комбин. объектов, ВНИИСИ, труды семинара. - М., 1985, с. 49-76.

5. Погорелов Б. А. Подметрики метрики Хемминга и теорема А. А. Маркова. - Труды по дискретной математике. Т. 9. - М: ФИЗМАТЛИТ, 2006, c. 190-219.

6. Погорелов Б. А., Пудовкина М. А. Подметрики метрики Хемминга и преобразования, распространяющие искажения в заданное число раз. Труды по дискретной математике. Т. 10. - М: ФИЗМАТЛИТ, 2007, c. 202-238.

7. Погорелов Б. А., Пудовкина М. А. Подметрики Хемминга и их группы изометрий. - Труды по дискретной математике. Т. 11. Вып. 2. - М: ФИЗМАТЛИТ, 2008, с. 147-191.

8. Сидельников B. М. Ассоциативные схемы и метрики на конечной группе. - Докл. РАН, 2004, т. 396, вып. 4, с. 455-459.

9. Сидельников B. М. Ассоциативные схемы и автоморфизмы конечных групп. - Матер. VIII Междунар. сем. «Дискретная математика и ее приложения». - М.: изд-во МГУ, 2004, с. 19-26.

10. Deza M. M., Deza E. Encyclopedia of Distances. - Springer-Verlag, 2009, p. 590. 